\title{
ANÁLISE DE ERROS PARA ALINHAMENTO EM AHRS - PARTE I: ALGORITMO TRIAD *
}

\author{
Lucas Pimenta Silva Paiva* \\ Fidelis Marcio Santos Rabelo de Melo Júnior* \\ Rogério Menezes Filho* Leonardo Alves Vieira* \\ Felipe Oliveira e Silva*
}

* Departamento de Automática, Universidade Federal de Lavras, MG, (e-mails: paiva.lucasps@gmail.com,fjunyn@gmail.com, rogeriofilho03@gmail.com, laviera1992@hotmail.com, felipe.oliveira@ufla.br).

\begin{abstract}
In most autonomous navigation systems, the initial orientation of the vehicle is not initially known. The alignment is a stage that precedes the navigation process, and is responsible for determining the orientation of the vehicle. However, low-grade inertial sensors are not recommended to perform the alignment process, since the readings of their angular rate sensors are not capable of providing accurate measurements of the Earth's rotation rate. Therefore, some authors propose the use of magnetometers, and the observation of the Earth's magnetic field density vector in the alignment. This paper investigates the problem of stationary alignment for low-grade Attitude and Heading Reference Systems (AHRS). In this work, we present the error analysis of the traditional Three-Axis Attitude Determination (TRIAD) algorithm. Simulated results corroborate the proposed errors analysis, evidencing the main differences, advantages and drawbacks of the use of each of these algorithms. Thus, this work serves as a basis for future research, especially those devoted to autonomous navigation using low-grade sensors.

Resumo: Na maioria dos sistemas de navegação autônoma, a orientação inicial do veículo não é conhecida inicialmente. $\mathrm{O}$ alinhamento é uma fase que antecede a etapa de navegação, e é responsável pela orientação do veículo. Contudo, sensores inerciais de baixo nível não são recomendados para realizar o processo de alinhamento, uma vez que as leituras de seus girômetros não são capazes de fornecer medições precisas da taxa de rotação terrestre. Diante disso, alguns autores propõem a utilização de magnetômetros, e utilizam observações do vetor densidade de campo magnético terrestre no alinhamento. Este artigo investiga o problema do alinhamento utilizando sensores de baixo nível para Sistemas de Referência de Orientação e Rumo (AHRS) em estado estacionário. Neste trabalho, é apresentada a análise de erros do tradicional algoritmo TRIAD (Determinação da Orientação via Três Eixos). Resultados simulados corroboram a análise de erros proposta. Dessa forma, este trabalho serve como base para futuras pesquisas, principalmente aquelas relacionadas à navegação autônoma utilizando sensores de baixo custo.
\end{abstract}

Keywords: Navigation System; Alignment; AHRS.

Palavras-chaves: Sistemas de Navegação; Alinhamento; AHRS.

\section{INTRODUÇÃO}

Um Sistema de Navegação Inercial (INS) é definido como um dispositivo capaz de fornecer posição, velocidade e orientação de um corpo, em um determinado sistema de coordenadas. Ele é, em geral, composto por uma Unidade de Medição Inercial (IMU) e um computador de bordo, responsável pela execução dos algoritmos embarcados.

\footnotetext{
‡ Esta pesquisa é financiada pela CAPES (Coordenação de Aperfeiçoamento de Pessoal de Nível Superior)(processo № 88881.169927/2018-01), Embrapa (processo № 212-20/2018) e Universidade Federal de Lavras (UFLA).
}

As IMUs, por sua vez, são compostas por um conjunto de acelerômetros e girômetros, capazes de medir variáveis tais como aceleração de força específica e taxa angular do corpo em movimento (Groves, 2008). A posição, velocidade e orientação do corpo são frutos da integração numérica das forças específicas e taxas angulares, medidas em instantes específicos de amostragem (Silva, 2016).

A configuração supracitada adquiriu relevância por volta do ano de 1960, sendo chamada de mecanização solidária, ou então, Sistema de Navegação Inercial Solidário (SINS), no qual os sensores estão firmemente montados no veículo (Titterton and Weston, 2004). 
Atualmente, verifica-se uma ampla utilização de IMUs na indústria, as quais se diferem por suas tecnologias de fabricação. Consequentemente, cada tipo de IMU possui diferentes desempenhos, originando uma relação diretamente proporcional entre precisão e custo. As IMUs podem ser classificadas em categorias como: marítimas, de aviação, intermediárias, táticas, automotivas e de consumo (Groves, 2008).

Como previamente mencionado, IMUs são utilizadas no cálculo da posição, velocidade e orientação de veículos. Isso se dá através da integração numérica das leituras fornecidas pelos acelerômetros e girômetros. Contudo, o processo de navegação requer uma inicialização prévia, mais conhecida como alinhamento, o qual consiste na estimação da orientação inicial do corpo com relação a uma referência conhecida (Silva, 2016). Os ângulos de Euler, matrizes de rotação e quaternions são os métodos mais utilizados para representar a orientação de um corpo. Ignorar o processo de inicialização pode desencadear erros inaceitáveis, resultando em grande perda de desempenho do SINS (Farrell, 2008).

$\mathrm{Na}$ maior parte dos casos, o alinhamento é realizado em estado estacionário, devido às condições particulares do veículo (Groves, 2008). Quando o próprio SINS do veículo define sua orientação em estado estacionário, dá-se o nome de auto-alinhamento (Britting, 1971) apud (Silva, 2016).

O auto-alinhamento é conduzido em duas fases, o autoalinhamento grosseiro (CA) e o auto-alinhamento fino (FA). A fase de auto-alinhamento grosseiro, como o próprio nome sugere, determina a orientação aproximada do veículo, por meio de transformações analíticas bastante diretas (Groves, 2008). As limitações deste método consistem em se considerar o sistema perfeitamente estacionário, os sensores não corrompidos e as informações externas precisamente conhecidas (Groves, 2008).

A despeito do CA ou FA, Gade (2016) afirma que é necessário uma entrada de referência para qualquer uma das etapas do alinhamento. Em casos estacionários, a orientação é definida através da observação de grandezas físicas existentes no globo terrestre, como a gravidade local e a taxa de rotação terrestre (Hu et al., 2004). Contudo, girômetros de baixo custo apresentam limitações para determinar a taxa de rotação da Terra, devido à sua pequena magnitude (Hu et al., 2004).

Diante disso, as medições de um magnetômetro podem ser muito úteis para se estimar e corrigir os erros em um SINS contendo sensores de baixo desempenho. Os magnetômetros, por exemplo, podem ser integrados com os girômetros, odômetros diferenciais, entre outros sensores, visando calibrar os biases dos mesmos, e também melhorar a precisão do alinhamento e da navegação (Groves, 2008). Na maior parte destas integrações, os acelerômetros são usados para determinação dos ângulos de rolamento e arfagem, enquanto magnetômetros são utilizados para corrigir o ângulo de guinada derivado dos girômetros ( $\mathrm{Li}$ and Wang, 2013). IMUs que incorporam magnetômetros em sua estrutura, são conhecidos como Sistemas de Referência de Orientação e Rumo (AHRS).

Farrell et al. (1966) estabeleceram o método de alinhamento grosseiro mais referido na literatura, o qual se tornou base para maioria dos trabalhos subsequentes. Este método estima a orientação do sistema através dos vetores gravidade e velocidade angular da Terra. Em 1971, Britting (1971) propôs um novo método de alinhamento grosseiro. Este estima a matriz de orientação a partir de três vetores linearmente independentes, gerados pelo produto vetorial dos vetores gravidade e velocidade angular da Terra. Por esse motivo, o método de Britting ficou conhecido como TRIAD (Determinação da Orientação via Três Eixos) (Silva, 2016). Este mesmo método, mais tarde, também viria a ser utilizado para o alinhamento em AHRS (Stirling et al., 2005).

Atualmente, encontram-se disponíveis na literatura, diversos métodos que buscam resolver o problema de alinhamento em AHRS (Wu et al., 2018; Yun et al., 2008). De forma generalizada, todos eles se baseiam na observação do campo magnético terrestre, via magnetômetros (Stirling et al., 2005; Yun et al., 2008; Wu et al., 2018).

Este artigo, por sua vez, tem como objetivo apresentar o modelo e análise de erros do algoritmo TRIAD, para o alinhamento em AHRS. Como principal contribuição deste trabalho, destaca-se a derivação analítica dos erros desenvolvidos no algoritmo TRIAD, uma vez que, na maioria dos trabalhos disponíveis na literatura, esses erros são apenas analisados e comparados via resultados numéricos.

O restante deste trabalho é organizado da seguinte forma: Na Seção 2, é descrito o algoritmo TRIAD. Na Seção 3 , é apresentada a análise de erros proposta. Resultados simulados são fornecidos na Seção 4, assim como a discussão sobre estes. Por fim, na Seção 5, encontram-se as conclusões desta pesquisa.

\section{ALINHAMENTO EM AHRS}

\subsection{Algoritmo Baseado na Determinação da Orientação via Três Eixos (TRIAD)}

O algoritmo TRIAD pode ser entendido como um método criado para a resolução do problema de Wahba (Wahba, 1965). Segundo Yun et al. (2008), o método TRIAD requer, como entrada para o sistema, a medição de dois vetores de referência não paralelos. No caso de AHRS, os vetores de entrada são observações da gravidade local, $\mathbf{g}_{P}$, fornecida pelos acelerômetros, e da densidade de campo magnético terrestre, $\mathbf{m}_{E}$, fornecida pelos magnetômetros (Jiang, 1998). No sistema de coordenadas de navegação $(\mathrm{NED})^{1}$, tem-se,

$$
\begin{gathered}
\mathbf{g}_{P}^{l}=\left[\begin{array}{lll}
0 & 0 & g_{P}
\end{array}\right]^{T}, \\
\mathbf{m}_{E}^{l}=B\left[\begin{array}{cll}
c \alpha c \gamma & s \alpha c \gamma & s \gamma
\end{array}\right]^{T},
\end{gathered}
$$

onde $g_{P}$ é a magnitude do vetor gravidade, $\alpha$ e $\gamma$ são ângulos de inclinação e declinação local do campo magnético da Terra, respectivamente e $B$ corresponde à magnitude da densidade de campo magnético.

Os valores acima podem ser representados no sistema de coordenadas do corpo ${ }^{2}$, assumindo que o AHRS é

\footnotetext{
1 Representado pelo sobrescrito $l$, e definido com seus eixos $x, y$ e $z$, apontados para norte, leste e down, respectivamente.

2 Representado pelo sobrescrito $b$, e definido com seus eixos $x, y$ e $z$, apontados para o eixo longitudinal do veículo, eixo transversal
} 
estacionário, e que os sensores não estão corrompidos. Sendo assim,

$$
\begin{gathered}
\mathbf{g}_{P}^{b} \approx-\mathbf{a}_{S F}^{b}=\left[\begin{array}{lll}
-a_{x} & -a_{y} & -a_{z}
\end{array}\right]^{T}, \\
\mathbf{m}_{E}^{b} \approx \mathbf{m}_{m}^{b}=\left[\begin{array}{lll}
m_{x} & m_{y} & m_{z}
\end{array}\right]^{T},
\end{gathered}
$$

onde $\mathbf{a}_{S F}$ é o vetor força específica, $a_{x}, a_{y}$ e $a_{z}$ são os valores medidos nos três eixos dos acelerômetros, $\mathbf{m}_{m}$ é o vetor de densidade total do fluxo magnético medido pelos magnetômetros, e $m_{x}, m_{y}$ e $m_{z}$ são os valores medidos nos eixos dos magnetômetros.

Segundo Zhao et al. (2011), o algoritmo constrói duas tríades de vetores, as quais são utilizadas para formar a matriz $C_{b}^{l}$, a saber,

$$
C_{b}^{l}=\left[\begin{array}{c}
\left(\mathbf{g}_{P}^{l}\right)^{T} \\
\left(\mathbf{m}_{E}^{l}\right)^{T} \\
\left(\mathbf{g}_{P}^{l} \times \mathbf{m}_{E}^{l}\right)^{T}
\end{array}\right]^{-1}\left[\begin{array}{c}
\left(\mathbf{g}_{P}^{b}\right)^{T} \\
\left(\mathbf{m}_{E}^{b}\right)^{T} \\
\left(\mathbf{g}_{P}^{b} \times \mathbf{m}_{E}^{b}\right)^{T}
\end{array}\right] .
$$

Os ângulos de rolamento $\phi$, arfagem $\theta$ e guinada $\psi$ podem ser encontrados através da relação,

$$
\begin{gathered}
\phi=\operatorname{atan}_{2} \frac{a_{y}}{a_{z}} \\
\theta=\operatorname{asin} \frac{a_{x}}{g_{p}} \\
\psi=\operatorname{atan}_{2} \frac{g_{P} m_{x} s \alpha-a_{y} m_{z} c \alpha+a_{z} m_{z} c \alpha+B a_{x} s \alpha s \gamma}{a_{y} m_{z} s \alpha-a_{z} m_{y} s \alpha+g_{P} m_{x} c \alpha+B a_{x} c \alpha s \gamma}
\end{gathered}
$$

\section{ANÁLISE DE ERROS}

A despeito da validade das equações apresentadas na Seção 2, elas se basearam nas seguintes premissas: veículo perfeitamente estacionário, sensores não corrompidos, e informações externas (gravidade e campo magnético terrestre) perfeitamente conhecidas. Segundo Hu et al. (2004), nenhuma das premissas supracitadas é verdadeira em aplicações reais. Por isso, na prática, o valor exato da matriz de rotação $C_{b}^{l}$ não pode ser determinado (Silva and Leite Filho, 2013).

Segundo Savage (2007), a existência de erros nas variáveis acima descritas produz uma matriz de rotação corrompida $\hat{\mathrm{C}}_{b}^{l}$, que é apenas uma aproximação da matriz $C_{b}^{l}$. A relação entre estas variáveis pode ser equacionada como,

$$
\hat{C}_{b}^{l}=C_{b}^{l}+\delta C_{b}^{l}=(I+E) C_{b}^{l},
$$

onde $I$ é a matriz identidade, e $E$ e $\delta C_{b}^{l}$ são matrizes representando os erros em $\hat{\mathrm{C}}_{b}^{l}$.

De acordo com Savage (2007), a matriz de erros $E$ é definida como,

$$
E=E_{s}+E_{s s}
$$

com,

$$
\begin{gathered}
E_{s}=\frac{E+E^{T}}{2}=\left[\begin{array}{lll}
\eta_{N} & o_{D} & o_{E} \\
o_{D} & \eta_{E} & o_{N} \\
o_{E} & o_{N} & \eta_{D}
\end{array}\right], \\
E_{s s}=\frac{E-E^{T}}{2}=\left[\begin{array}{rrr}
0 & \varphi_{D} & -\varphi_{E} \\
-\varphi_{D} & 0 & \varphi_{N} \\
\varphi_{E} & -\varphi_{N} & 0
\end{array}\right],
\end{gathered}
$$

onde $E_{s}$ é uma matriz simétrica e $E_{s s}$ é uma matriz antisimétrica. Os vetores de erros são definidos como: o (erro

direito, e eixo transversal completando a regra da mão direita, respectivamente. de ortogonalidade), $\eta$ (erro de normalidade) e $\varphi$ (erro de alinhamento) ${ }^{3}$.

Manipulando-se (9), tem-se que a matriz $E$ também pode ser representada como,

$$
E=\delta C_{b}^{l}\left(C_{b}^{l}\right)^{T}
$$

Segundo Silva (2016), pode-se investigar os erros desenvolvidos em $C_{b}^{l}$ através de técnicas de perturbação linear. Dessa forma, o autor descreve $\delta C_{b}^{l}$ como,

$$
\begin{aligned}
\delta C_{b}^{l}=\frac{\delta C_{b}^{l}}{\delta a_{x}} \delta a_{x}+\frac{\delta C_{b}^{l}}{\delta a_{y}} \delta a_{y}+\frac{\delta C_{b}^{l}}{\delta a_{z}} \delta a_{z} \\
+\frac{\delta C_{b}^{l}}{\delta m_{x}} \delta m_{x}+\frac{\delta C_{b}^{l}}{\delta m_{y}} \delta m_{y}+\frac{\delta C_{b}^{l}}{\delta m_{z}} \delta m_{z} \\
+\frac{\delta C_{b}^{l}}{\delta g_{P}} \delta g_{P}+\frac{\delta C_{b}^{l}}{\delta \alpha} \delta \alpha+\frac{\delta C_{b}^{l}}{\delta \gamma} \delta \gamma+\frac{\delta C_{b}^{l}}{\delta B} \delta B
\end{aligned}
$$

onde, $\delta_{x}$ representa o erro na variável genérica $x$.

Os erros de normalidade, ortogonalidade e alinhamento, portanto, podem ser computados através da utilização de (5) e (14) em (13), e do resultado em (10) a (12).

De acordo com Silva (2016), fórmulas para os erros nos ângulos de Euler também podem ser derivadas substituindose $C_{b}^{l}$ em (14), respectivamente por $\phi, \theta$ e $\psi$. Ainda segundo Silva (2016), as expressões obtidas através do procedimento supracitado, para o caso genérico de um veículo arbitrariamente orientado com relaçao ao sistema de navegação, são complexas e de difícil interpretação. Sendo assim, o autor adota a condição de perfeito alinhamento entre os triedros do corpo e de navegação em seu trabalho, premissa essa que também é adotada nesse artigo.

Para determinação analítica dos erros provenientes do método TRIAD, utiliza-se (5) em (14), e o resultado em (10) a (12). Dessa forma, as expressões que descrevem os erros residuais do algoritmo podem ser descritas como,

$$
\begin{aligned}
\eta_{N}=\frac{c \alpha t \gamma}{g_{P}} & \delta a_{x}+\frac{c \alpha t \gamma}{g_{P}} \delta a_{y}-\frac{s^{2} \alpha}{g_{P}} \delta a_{z}+\frac{c \alpha}{B c \gamma} \delta m_{x} \\
+ & \frac{s \alpha}{B c \gamma} \delta m_{y}-\frac{s^{2} \alpha}{g_{P}} \delta g_{P}+t \gamma \delta \gamma-\frac{1}{B} \delta B
\end{aligned}
$$

$$
\begin{aligned}
\eta_{E}=\frac{c \alpha t \gamma}{g_{P}} \delta & a_{x}+\frac{c \alpha t \gamma}{g_{P}} \delta a_{y}-\frac{s^{2} \alpha}{g_{P}} \delta a_{z}+\frac{c \alpha}{B c \gamma} \delta m_{x} \\
+ & \frac{s \alpha}{B \gamma} \delta m_{y}-\frac{c^{2} \alpha}{g_{P}} \delta g_{P}+t \gamma \delta \gamma-\frac{1}{B} \delta B
\end{aligned}
$$$$
\eta_{D}=-\frac{1}{g_{P}} \delta a_{z}-\frac{1}{g_{P}} \delta g_{P}
$$

$$
\begin{aligned}
o_{N}= & \frac{s(2 \gamma)}{4 g_{P}} \delta a_{x}-\frac{s^{2} \alpha}{2 g_{P}} \delta a_{y}+\frac{s \alpha t \gamma}{2 g_{P}} \delta a_{z} \\
& +\frac{s \alpha}{2 B c \gamma} \delta m_{z}+\frac{s \alpha t \gamma}{2 g_{P}} \delta g_{p}-\frac{s \alpha}{2} \delta \gamma-\frac{s \alpha t \gamma}{2 B} \delta B,
\end{aligned}
$$

\footnotetext{
3 Subscritos $N, E$ e $D$ representam erros nas direções norte, leste e down, respectivamente
} 


$$
\begin{aligned}
& o_{E}=-\frac{c^{2} \alpha}{2 g_{P}} \delta a_{x}-\frac{s(2 \alpha)}{4 g_{P}} \delta a_{y}+\frac{c \alpha t \gamma}{2 g_{P}} \delta a_{z} \\
& +\frac{c \alpha}{2 B c \gamma} \delta m_{z}+\frac{c \alpha t \gamma}{2 g_{P}} \delta g_{p}-\frac{c \alpha}{2} \delta \gamma-\frac{c \alpha t \gamma}{2 B} \delta B, \\
& o_{D}=\frac{s(2 \alpha)}{2 g_{P}} \delta a_{z}+\frac{s(2 \alpha)}{2 g_{P}} \delta g_{P}, \\
& \varphi_{N}=\frac{s(2 \gamma)}{4 g_{P}} \delta a_{x}+\frac{c^{2} \alpha+1}{2 g_{P}} \delta a_{y}+\frac{s \alpha t \gamma}{2 g_{P}} \delta a_{z} \\
& +\frac{s \alpha}{2 B c \gamma} \delta m_{z}+\frac{s \alpha t \gamma}{2 g_{P}} \delta g_{p}-\frac{s \alpha}{2} \delta \gamma-\frac{s \alpha t \gamma}{2 B} \delta B, \\
& \varphi_{E}=\frac{s^{2} \gamma+1}{2 g_{P}} \delta a_{x}+\frac{s(2 \alpha)}{4 g_{P}} \delta a_{y}-\frac{c \alpha t \gamma}{2 g_{P}} \delta a_{z} \\
& -\frac{c \alpha}{2 B c \gamma} \delta m_{z}-\frac{c \alpha t \gamma}{2 g_{P}} \delta g_{p}-\frac{c \alpha}{2} \delta \gamma+\frac{c \alpha t \gamma}{2 B} \delta B, \\
& \varphi_{D}=\frac{s \alpha t \gamma}{g_{P}} \delta a_{x}+\frac{c \alpha t \gamma}{g_{P}} \delta a_{y} \\
& -\frac{s \alpha}{B c \gamma} \delta m_{x}+\frac{c \alpha}{B c \gamma} \delta m_{y}-\delta \alpha, \\
& \delta \phi=-\frac{1}{g_{P}} \delta a_{y}, \\
& \delta \theta=\frac{1}{g_{P}} \delta a_{x}, \\
& \delta \psi=\frac{s \alpha t \gamma}{g_{P}} \delta a_{x}-\frac{c \alpha t \gamma}{g_{P}} \delta a_{y}+\frac{s(2 \alpha)}{2 g_{P}} \delta a_{z} \\
& +\frac{s \alpha}{2 B c \gamma} \delta m_{x}+\frac{c \alpha}{2 B c \gamma} \delta m_{y}+\frac{s(2 \alpha)}{2 g_{P}} \delta g_{P}+\delta \alpha \text {. }
\end{aligned}
$$

A análise de erros é proposta para verificar a influência dos fatores externos no processo de alinhamento em AHRS. Com base em (15) a (20), nota-se que erros residuais de normalidade e ortogonalidade são desenvolvidos na $C_{b}^{l}$.

Apesar da existência dos erros de normalidade e ortogonalidade, os erros de alinhamento são de maior preocupação, uma vez que eles não são adequadamente estimados e compensados nem mesmo na etapa posterior de alinhamento fino. Como pode ser observado em (21) a (23), os erros de alinhamento são afetados pela maioria das fontes de erros investigadas neste trabalho (Silva et al., 2017). Nota-se, em (26), que estas fontes de erro também afetam o erro de guinada.

Verifica-se também que, nas equações supracitadas, os componentes de erros ponderados por $s \alpha$ são menos prejudiciais para o desempenho do alinhamento, uma vez que $\alpha$ normalmente se situa entre $\pm 20^{\circ}$ para a maioria das aplicações terrestres (Chulliat et al., 2015). Contudo, é possível inferir, de (21), que embora $s \alpha$ pondere o valor de $\delta m_{z}$, seu efeito pode ser significativo no erro de alinhamento norte $\varphi_{N}$, especialmente na presença do chamado magnetismo "hard-iron"(Groves, 2008).

Por último, vale a pena notar que $t \gamma$ gera erros insignificantes em baixas latitudes. Em contrapartida, esse erro aumenta significativamente à medida que a latitude aumenta, causando erros residuais consideráveis no alinhamento. Esta, portanto, é a principal razão pela qual o alinhamento de AHRS não é viável nos pólos geográficos da Terra.

\section{RESULTADOS SIMULADOS}

Para validar a análise de erros apresentada na Seção 3, bem como as verificações descritas, foi elaborado um teste simulado.

Os resultados se baseiam no teste de desempenho do processo de alinhamento usando o algoritmo TRIAD. Para esta simulação, foram considerados valores da latitude local $-23^{\circ} 12^{\prime} 47^{\prime \prime}$, longitude $-45^{\circ} 51^{\prime} 38^{\prime \prime}$ e altitude 629 metros. Os dados da Terra, como raio equatorial, elipticidade, velocidade angular, entre outros, foram baseados no trabalho de Rogers (2007) apud Silva (2016). A aceleração da gravidade local e o campo magnético da Terra foram calculados de acordo com os modelos descritos em Britting (1971) e Chulliat et al. (2015), respectivamente.

Por convenção, as simulações foram concebidas assumindose que o sistema de coordenadas do corpo se encontra perfeitamente alinhado com o sistema de coordenadas de navegação, representando, portanto, um cenário ideal.

Os sensores (acelerômetros e magnetômetros) foram simulados estacionários, com frequência de amostragem de 100 Hz. Os biases simulados dos magnetômetros e acelerômetros foram de, respectivamente, $5 \mathrm{mG}$ e $5 \mathrm{mg}$. Também foram utilizados valores de random walk para os acelerôme$\operatorname{tros}(0.1 \mathrm{mg} / \sqrt{\mathrm{Hz}})$ e magnetômetros $(0.2 \mathrm{mG} / \sqrt{\mathrm{Hz}})$. Incertezas nos valores da gravidade local, ângulo de declinação, ângulo de inclinação, e magnitude do campo magnético local também foram consideradas, a saber, $0,005 \mathrm{mg}, 0.1^{\circ}$, $0,1^{\circ}$ e $0,1 \mathrm{mG}$, respectivamente. Esses valores simulam uma Unidade de Medição Inercial de nível automotivo conforme as especificações descritas no trabalho de Groves (2008).

Com base nos erros simulados foi possível obter estimativas para os erros de normalidade, ortogonalidade, alinhamento e ângulos de Euler. Esses valores são oriundos das fórmulas apresentadas na Seção 3, e estão representados na Tabela 1.

A Figura 1, por outro lado, ilustra os erros obtidos ao longo do processo de alinhamento. Como esperado, o resultado do alinhamento via algoritmo TRIAD resultou em erros na matriz de rotação computada (erros de normalidade,

Tabela 1. Erros simulados.

\begin{tabular}{cc}
\hline Erros & TRIAD \\
\hline$\eta_{N}\left[^{\circ}\right]$ & 0,6451 \\
$\eta_{E}\left[^{\circ}\right]$ & 0,4235 \\
$\eta_{D}\left[^{\circ}\right]$ & $-0,2873$ \\
$\mathrm{o}_{N}\left[^{\circ}\right]$ & $-0,2070$ \\
$\mathrm{o}_{E}\left[^{\circ}\right]$ & 0,5196 \\
$\mathrm{o}_{D}\left[^{\circ}\right]$ & $-0,1049$ \\
$\varphi_{N}\left[^{\circ}\right]$ & 0,0781 \\
$\varphi_{E}\left[^{\circ}\right]$ & $-0,8140$ \\
$\varphi_{D}\left[^{\circ}\right]$ & 1,6594 \\
$\delta \phi\left[^{\circ}\right]$ & $-0,2903$ \\
$\delta \theta\left[^{\circ}\right]$ & 0,2855 \\
$\delta \psi\left[^{\circ}\right]$ & $-1,7400$ \\
\hline Fonte: Autor.
\end{tabular}



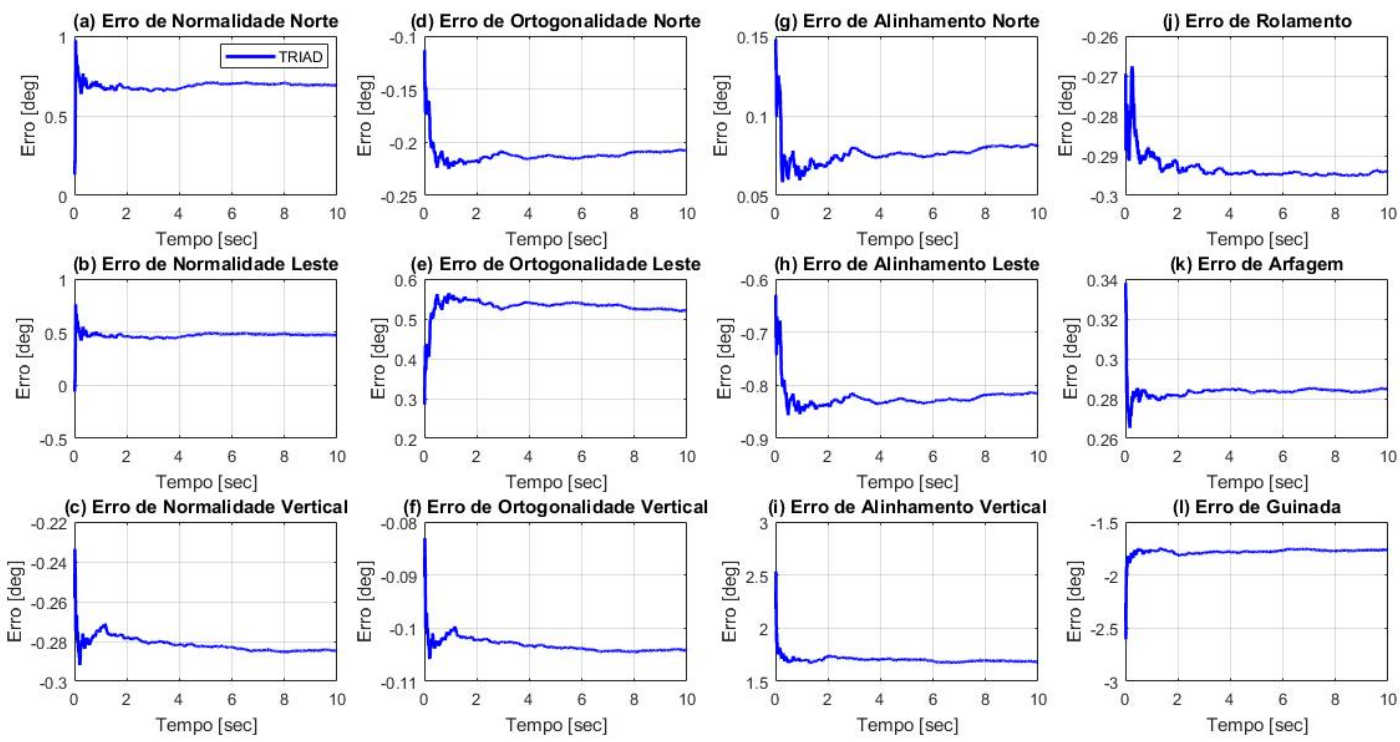

Figura 1. Erros Simulados

Fonte: Autor.

ortogonalidade e alinhamento), bem como nos ângulos de Euler.

Verifica-se também que os erros de alinhamento vertical e de guinada foram os mais expressivos, isto é, apresentaram maior magnitude com relação aos demais. Isso pode ser justificado com base em (26), onde pode-se verificar que as variáveis supracitadas dependem de uma maior quantidade de fontes de erro.

Por fim, conclui-se que o algoritmo TRIAD foi capaz de estimar a orientação do corpo nos três eixos, apresentando um bom desempenho no alinhamento em AHRS. Como pode-se verificar, os erros obtidos (Figura 1) são compatíveis com os erros preditos (Tabela 1). Estes resultados, portanto, confirmam a validade da análise de erros proposta neste artigo.

\section{CONCLUSÕES}

Neste artigo, o algoritmo de alinhamento grosseiro TRIAD, para Sistemas de Referência de Orientação e Rumo (AHRS) estacionários, foi revisado. Uma análise de erros detalhada e abrangente foi proposta, considerando a hipótese ideal dos triedros de navegação e do corpo perfeitamente alinhados.

A análise de erros proposta levou em consideração erros nas leituras dos acelerômetros e magnetômetros, além de incertezas nos modelos gravitacional e magnético da Terra. Sendo assim, foi apresentada a formulação analítica dos erros desenvolvidos na matriz de rotação (erros de normalidade, ortogonalidade e alinhamento), como também nos ângulos de Euler.

Com base nas expressões obtidas, foi possível investigar o algoritmo TRIAD, identificando os erros que mais afetam seu desempenho. Notou-se que o algoritmo TRIAD é corrompido por todos os tipos de erros analisados. Ele também apresenta maior susceptibilidade aos erros de ali- nhamento vertical e erro de guinada, os quais são, em geral, mais prejudiciais à navegação, uma vez que o alinhamento fino não é capaz de compensá-los efetivamente. De forma geral, estes erros são causados por incertezas nas leituras dos acelerômetros e, principalmente, dos magnetômetros.

Foi possível verificar, também, que os erros nos ângulos de rolamento e arfagem são afetados exclusivamente pelos biases dos acelerômetros. Os erros de alinhamento norte e leste, por outro lado, são influenciados pela leitura do magnetômetros no eixo $z$, pelo erro do ângulo de inclinação magnética, pelo erro na gravidade local, e pelo erro na magnitude da densidade de fluxo magnético da Terra.

Por fim, como sugestão para trabalhos futuros, tem-se, para fins de comparação, a análise de erros de outros algoritmos de alinhamento em AHRS, descritos na literatura. Dentre eles, pode-se citar: o Algoritmo Baseado em Ângulos de Euler (EBA), o Algoritmo Quaternion Fatorado (FQA), e o Algoritmo Quaternion Algébrico (AQUA). Adicionalmente, também pretende-se investigar como a análise de erros proposta neste trabalho pode ser extendida para o cenário genérico dos sistemas de coordenadas do corpo e de navegação arbitrariamente orientados, como também validar a análise com os testes experimentais. Dessa forma será possível obter dados para análise e comparação do desempenho de diferentes algoritmos de alinhamento em AHRS.

\section{AGRADECIMENTOS}

Os autores agradecem à ao Departamento de Automática (DAT) da Universidade Federal de Lavras (UFLA), pelo suporte dado à pesquisa.

\section{REFERÊNCIAS}

Britting, K.R. (1971). Inertial navigation systems analysis. Chulliat, A., Macmillan, S., Alken, P., Beggan, C., Nair, M., Hamilton, B., Woods, A., Ridley, V., Maus, S., and 
Thomson, A. (2015). The US/UK world magnetic model for 2015-2020.

Farrell, J. (2008). Aided navigation: GPS with high rate sensors. McGraw-Hill, Inc.

Farrell, J., Knight, J., and Thompson, E. (1966). Alignment methods for strapdown inertial systems. Journal of Spacecraft and Rockets, 3(9), 1432-1434.

Groves (2008). Principles of GNSS, inertial, and multisensor integrated navigation systems.

Hu, X., Liu, Y., Wang, Y., and Yan, D. (2004). Errors analysis and compensation of low cost attitude measurement system. In Intelligent Control and Automation, 2004. WCICA 2004. Fifth World Congress on, volume 4, 3688-3691. IEEE.

Jiang, Y.F. (1998). Error analysis of analytic coarse alignment methods. IEEE Transactions on Aerospace and Electronic Systems, 34(1), 334-337.

Li, W. and Wang, J. (2013). Effective adaptive Kalman filter for MEMS-IMU/magnetometers integrated attitude and heading reference systems. The Journal of Navigation, 66(1), 99-113.

Savage, P. (2007). Strapdown analytics: Part 1, maple plain, mn: Strapdown associates.

Silva, F.O. (2016). Stationary Self-Alignment and $\mathrm{Ca}$ libration Techniques for Straodown Inertial Navigation Systems. Addison-Wesley.

Silva, F.O., Hemerly, E.M., and Leite Filho, W.C. (2017). On the error state selection for stationary sins alignment and calibration kalman filters - part ii: Observability/estimability analysis. Sensors, 17(3), 439.

Silva, F.O. and Leite Filho, W.C. (2013). Análise do tempo de alinhamento em sistemas de navegação inercial solidários. Proceeding Series of the Brazilian Society of Computational and Applied Mathematics, 1(1).

Stirling, R., Fyfe, K., and Lachapelle, G. (2005). Evaluation of a new method of heading estimation for pedestrian dead reckoning using shoe mounted sensors. The Journal of Navigation, 58(1), 31-45.

Titterton, D. and Weston (2004). Strapdown inertial navigation technology, volume 17. IET.

Wahba, G. (1965). A least squares estimate of spacecraft attitude. SIAM Review, 7(3), 409.

Wu, J., Zhou, Z., Fourati, H., and Cheng, Y. (2018). A super fast attitude determination algorithm for consumerlevel accelerometer and magnetometer. IEEE Transactions on Consumer Electronics, 64(3), 375-381.

Yun, X., Bachmann, E.R., and McGhee, R.B. (2008). A simplified quaternion-based algorithm for orientation estimation from earth gravity and magnetic field measurements. IEEE Transactions on instrumentation and measurement, 57(3), 638-650.

Zhao, H., Shang, H., Wang, Z., and Jiang, M. (2011). Comparison of initial alignment methods for SINS. In Intelligent Control and Automation (WCICA), 2011 9th World Congress on, 42-47. IEEE. 